\title{
Belief Elicitation with a Synchronized Lottery Choice Menu that is Invariant to Risk Attitudes: Online Appendix
}

Charles A. Holt and Angela M. Smith

\section{Common Instructions Across All Treatments - Pages 1-2}

Rounds: The experiment consists of a sequence of "rounds".

Guessing Which Cup is Used: In each round, we will use the computer to simulate one or more draws of a colored marble from a "cup". There are two cups, with different mixes of colored marbles. You will be asked to assess the "chances out of 100" that a particular cup is being used. [For the QSR-numbers treatment, the previous sentence was replaced by: You will be asked to make a decision based on how likely it is that a particular cup is being used.]

Choosing Which Cup to Use: First, we draw a computer-generated random number, which will be either $1,2, \ldots 6$. Think of this as the throw of a die with 6 sides, with each side being equally likely.

* If the roll of the die yields 1 - 3, then the draw will be from the Blue cup, which contains more blue marbles.

* If the roll of the die yields 4 - 6, then the draw will be from the Red cup, which contains more red marbles.

Therefore, it is equally likely that either cup will be selected.

\section{Blue Cup}

Used if the die throw is $1-3$

Contents:

B

2 blue marbles and 1 red marble

\section{Red Cup}

Used if the die throw is 4 - 6

Contents: $\quad \mathrm{R} \quad \mathrm{R} \quad \mathrm{B}$ 2 red marbles and 1 blue marble

Colored marbles: The contents of each cup are shown above. 
Cup Used: You will not be told in advance the result of the die throw, so you will not know which cup is being used. The computerized die is thrown separately for each person for each round, so the cup that is used for you may or may not be the same as the cup being used for another person.

Private Draws: Once the computerized die throw determines the cup to be used, you may be shown one or more randomly selected marbles drawn from that cup. These draws (if any) may provide information to you about which cup is being used for your draws, but they will not provide any information about the cups being used for others, since the cup used may differ from person to person.

Independent Draws: In some rounds you may see more than one draw from the same cup. You will see them in the order that they are drawn. The draws will be done independently, so it is as if we were to shake the cup, pick a marble at random, show it to you, return it to the cup, and shake it again before drawing another randomly selected marble, so the contents of the cup are always the same when a draw is made.

Draws Made with Replacement: After each draw is made, it is as if the marble is returned to the cup before the next draw is made. Therefore, in some rounds you might see more than 3 draws, even though there are only 3 marbles in the cup being used!

\section{LC Treatment Instructions (pages 3-6)}

After you have seen the draws (if any) for the current round, you with be given a series of choices between a lottery that pays a reward of $\mathbf{\$ 2 . 0 0}$ if the Red Cup is being used in the current round and a lottery that pays a reward of $\mathbf{\$ 2 . 0 0}$ with a specified probability ("chances out of 100") that does not depend on which cup is being used.

For example, the top row of the table below offers a choice between " 0 chances out of $100 "$ of $\$ 2.00$ (left side) and a payment of $\$ 2.00$ if the Red Cup is in fact being used (right side). Thus the payoff for the Random Lottery on the left is zero for sure, so you would want to choose the Red Cup lottery on the right, and this decision has been entered as a default in the top row.

The decision for the bottom row would also be straightforward, since it involves a sure payment of $\$ 2.00$ ("100 chances out of 100") on the left side and a payment of $\$ 2.00$ to 
be made only if the Red Cup is being used on the right side. Hence the default choice for the sure payment on the left has been entered in the bottom row.

Your choice for the middle row would depend on which cup is more likely to be the one that is being used, based on the $\operatorname{draw}(\mathrm{s})$ for the current round. If you do think the Red Cup is more likely, i.e. that it provides more than 50 chances out of 100 of the high payoff, then you should choose the Red Cup Lottery on the right side. Conversely, you should choose the Random Lottery in the middle on the left side (with 50 chances in 100 of the high payoff), if you think the Blue Cup is more likely.

The decision table to be used will have more than 3 rows, and it will be constructed so that your best chance of obtaining \$2.00 is achieved switching from the Red Cup Lottery on the right to a Random Lottery at a row that corresponds to your true beliefs about the chances that the Red Cup is being used, as explained next.

\section{Random Lottery}

0 chances in 100 of $\$ 2.00$.

50 chances in 100 of $\$ 2.00$

100 chances in 100 of $\$ 2.00^{\mathbb{C}}$

\section{Red Cup Lottery}

⿷ $\$ 2.00$ if the Red Cup was used

\$2.00 if the Red Cup was used . \$2.00 if the Red Cup was used

Payoff Table: You will be given a table with a number of decision rows, as shown below. After you make a choice for each of the rows, one row will be selected (at random) to determine your payoff, which will depend on whether you chose the Random Lottery or the Red Cup Lottery for that row. Thus if you choose the Red Cup lottery for the row that ends up being used, then you earn \$2.00 if the Red Cup was in fact used for that round. If you chose the Random Lottery for the row that ends up being used, then the Random Lottery will determine your payoffs, regardless of which cup was used to generate the draw(s) you saw.

Example: For example, if the draws you see cause you to conclude that the Red Cup is no more or less likely than the Blue Cup, then you would be indifferent the two options 
in the middle row below, with 50 chances of 100 of $\$ 2.00$ on the left side. In this case you would prefer the Red Cup lottery (on the right side) for all rows in the top part of the table, and you would prefer the Random lottery (on the left side) with more than 50 chances out of 100 for all rows in the bottom half of the table, as indicated by the decisions that have been marked. Even if you are indifferent (e.g. in the middle row below), you will have to select one of the two options in each row.

Random Lottery
0 chances in 100 of $\$ 2.00$.
10 chances in 100 of $\$ 2.00$
20 chances in 100 of $\$ 2.00$
30 chances in 100 of $\$ 2.00$

Example: Suppose that you choose the Red Cup Lottery over a Random Lottery with 40 chances in 100 of the high payoff, and you switch sides in the next line, choosing the Random Lottery with 50 chances in 100 of the high payoff over the Red Cup Lottery. This pattern reveals that you believe that the chances in 100 of the Red Cup being used are greater than or equal to 40 and less than or equal to 50 . 
More Precise Choices: For this "crossover row", you will see a drop-down menu to let you be more precise about exactly where you would switch from the Red Cup lottery on the right side to the Random Lottery on the left side. Please click on the select menu in the table below to see how this process will work.

Row Selection: Rows will be numbered: $\mathbf{1}$ for the row with 10 chances in 100 of the high payoff on the left, 2 for the row with 20 chances in 100, .., $\mathbf{1 0}$ for the row with 100 chances in 100. One of these 10 rows will be selected at random to be relevant ex post (the 0 row will not be used). If the "crossover" row is selected, we will generate another random number from 1 to 10 to determine which of the 10 rows referenced in the dropdown menu for that row is used.

\author{
Random Lottery \\ 0 chances in 100 of $\$ 2.00$. \\ 10 chances in 100 of $\$ 2.00$ \\ 20 chances in 100 of $\$ 2.00$ \\ 30 chances in 100 of $\$ 2.00^{-}$ \\ 40 chances in 100 of $\$ 2.00^{-}$ \\ Switch to Random Lottery if it offers at least: \\ 60 chances in 100 of $\$ 2.00^{\mathbb{C}}$ \\ 70 chances in 100 of $\$ 2.00^{\mathbb{C}}$ \\ 80 chances in 100 of $\$ 2.00^{\mathbb{C}}$ \\ 90 chances in 100 of $\$ 2.00^{\mathbb{C}}$ \\ 100 chances in 100 of $\$ 2.00^{\mathbb{C}}$

\section{Red Cup Lottery} \\ [2.00 if the Red Cup was used \\ E $\$ 2.00$ if the Red Cup was used \\ [2.00 if the Red Cup was used \\ [ $\$ 2.00$ if the Red Cup was used \\ [ $\$ 2.00$ if the Red Cup was used \\ C $\$ 2.00$ if the Red Cup was used \\ C \$2.00 if the Red Cup was used \\ C2.00 if the Red Cup was used \\ C \$2.00 if the Red Cup was used \\ .\$2.00 if the Red Cup was used
}

\title{
Summary (Final Page of Instructions)
}




\section{Blue Cup Red Cup}

(if die throw is 1 - 3) (if die throw is 4 - 6)

\section{B B R $\quad$ R R B}

Cup Used: If the roll of the die yields 1 - 3, then the draw will be from the Blue cup, which contains more blue marbles. If the roll of the die yields $4-6$, then the draw will be from the Red cup, which contains more red marbles. Note that each cup is equally likely to be used.

Information: After the cup to be used has been randomly determined, you will be shown sample draws, if any, from that cup (made with replacement) for the current round. After considering the available information, you enter your decisions for each row of the table provided.

Payoff Method: In each row of your decision table, you will choose between two alternative procedures for determining whether you would receive the higher payoff \$2.00 for that row. On the left side is the Random Lottery with a specified "chances in $100 "$ of getting the high payoff, and on the right side is the option that generates the higher payoff if the Red Cup is used. Coming down the table, your choices should switch from the right to the left side at the point where the chances in 100 for the Random Lottery exceed the chances in 100 that you think the Red Cup is being used.

Helpful Hint: Each row is equally likely to be used to determine your earnings, so you should think carefully about which decision in each row provides you with the highest chance of obtaining the best payoff.

Final Hint: After you look at the draws (if any) for a round, try to figure out which cup, Red or Blue, is more likely, and be sure that your decision reflects that intuition, i.e. that the chances in 100 for the Red Cup are greater than 50 if you think the Red Cup is more likely and less than 50 otherwise. 


\section{BDM Treatment Instructions (pages 3-6)}

When you have seen the draws (if any), you must decide on the "chances out of 100" that the cup being used is the Red Cup. If this is a round where no draws are made, you will have to base your decision completely on how the die throw determines the cup used. Otherwise you may use both information about how the cup is selected and about the specific draw or draws, keeping in mind the contents of the cups.

A decision of 0 means that you think there is no chance that the Red Cup is being used, a decision of 100 means that you are absolutely sure that the Red Cup is being used, a decision of 50 means that you think it is equally likely that each cup is being used, etc.

So choose a number above 50 if you think that it is more likely that the Red Cup is being used, and choose a number below 50 if you think it is more likely that the Blue Cup is being used.

Your decision (reported chances in 100 for the Red Cup) must be an integer between 0 and 100. Your earnings will be calculated in a manner such that your best chance of

obtaining a high earnings amount is achieved if you report your true beliefs about the chances in 100 that the Red Cup is being used, as explained next.

Payment Methods: There are two possible payoffs, a high payoff of $\mathbf{\$ 2 . 0 0}$, and a low payoff of $\mathbf{\$ 0 . 0 0}$, and there are two alternative methods that will be used to decide whether you receive the higher payoff.

Red Cup Payoff Method: One method will pay you the higher payoff if the Red Cup turns out to be the one that was used; you will receive the lower payoff if the cup used is the Blue Cup. This may not be an attractive option for you if the draws suggest that the Blue Cup is highly likely, so we offer an alternative.

"N Lottery" Payoff Method: The alternative method will be to use a lottery that has $\mathbf{N}$ chances out of $\mathbf{1 0 0}$ of providing the high payoff, where the exact number $\mathrm{N}$ is a randomly generated number between 0 and 100. Each value of $\mathrm{N}$ in this range is equally likely, so the $\mathrm{N}$ Lottery will be undesirable if $\mathrm{N}$ is low and it will desirable if $\mathrm{N}$ is high. The way the $\mathrm{N}$ lottery is played is to obtain a second random number, say $\mathrm{B}$, that equally likely to be any one of 100 integers: $0,1, \ldots$ 99. If the second number is less than $\mathrm{N}$, then 
the $\mathrm{N}$ lottery yields the higher payoff. So if $\mathrm{N}=1$, there is a 1 in 100 chance of getting the high payoff, if $\mathrm{N}=2$ there is a 2 in 100 chance, etc.

Choice of Payoff Method: The computer will use your reported chances of 100 for the red cup to select the payoff method that offers you the best chance of earning the high payoff, as explained next.

Choice of Payoff Method (next page of instructions): After you make your decision, the computer will randomly determine the value of $\mathrm{N}$ : if $\mathrm{N}$ is greater than your decision, then on average the $\mathrm{N}$ lottery will give you a greater chance of the high payoff, and therefore, it will be used. If your decision is greater than or equal to $\mathrm{N}$, however, you have deemed the chances in 100 for the Red Cup to be high enough so the red cup lottery is best for you.

Making Your Decision: In making your decision, you should think carefully about the chances out of 100 that the Red Cup is being used, since making a good decision based on the available information will help the computer select that payoff method that is best for you. If your decision represents your true beliefs about the chances that the red cup is being used, then the computer will select the payment method that provides you with the best chance of the high payoff.

\section{Summary (Final Page of Instructions)}

\section{Blue Cup $\quad$ Red Cup}

(if die throw is 1 - 3) (if die throw is 4 - 6)

\section{B B R R R B}

Cup Used: If the roll of the die yields 1 - 3, then the draw will be from the Blue cup, which contains more blue marbles. If the roll of the die yields 4 - 6, then the draw will be from the Red cup, which contains more red marbles. Note that each cup is equally likely to be used.

Decision: At the start of each round, you will see the draws, if any, (with replacement) for the current round. After considering the available information, you will enter your decision (an integer between 0 and 100) in the box next to the Submit button.

Payoff Method: The two possible payoffs in each round are: $\$ 2.00$ and $\mathbf{\$ 0 . 0 0}$. 
Payoff Method: There is an N Lottery that has N chances in 100 of providing the higher payoff. There is a Red Cup Lottery that provides the higher payoff if the Red Cup is used. The computer will select the best payoff method based on your assessment (P) of the chances in 100 that the Red Cup is being used this round. In particular, the $\mathrm{N}$ lottery will only be relevant if it provides a greater chance of the high payoff.

Helpful Hint: Your decision should be your best guess about the chances out of 100 that the Red Cup is being used, so that the computer can select the payoff option, $\mathrm{N}$ Lottery or Red Cup Lottery, that is best for you.

Final Hint: After you look at the draws (if any) for a round, try to figure out which cup, Red or Blue, is more likely, and be sure that your decision reflects that intuition, i.e. that the chances in 100 for the Red Cup are greater than 50 if you think the Red Cup is more likely and less than 50 otherwise.

\section{QSR-chances Treatment Instructions (pages 3-6)}

When you have seen the draws (if any), you must decide on the "chances out of 100" that the cup being used is the Red Cup. If this is a round where no draws are made, you will have to base your decision completely on how the die throw determines the cup used. Otherwise you may use both information about how the cup is selected and about the specific draw or draws, keeping in mind the contents of the cups.

A decision of 0 means that you think there is no chance that the Red Cup is being used, a decision of 100 means that you are absolutely sure that the Red Cup is being used, a decision of 50 means that you think it is equally likely that each cup is being used, etc.

So choose a number above 50 if you think that it is more likely that the Red Cup is being used, and choose a number below 50 if you think it is more likely that the Blue Cup is being used.

Your decision (reported chances in 100 for the Red Cup) must be an integer between 0 and 100. If you were to divide your decision by 100, the ratio can be thought of as a probability that the Red Cup is being used. Your payoff will be a base amount $\$ 2.00$ minus a penalty that depends on how much probability you assign to the cup, Red or Blue, that is NOT used. The formula is constructed to provide you with an incentive to 
report your true beliefs about the chances that the Red Cup is being used. The exact formula doesn't matter, since the calculations will be done for you, as explained on the next page.

Payment Method: After you have submitted your "chances in 100" that the Red Cup is being used, you will be informed about which cup was actually used. You will earn more money to the extent to which your decision indicated higher chances in 100 for the cup that turned out to be the one that actually was used. Your earnings for the round will be between a high payoff of $\mathbf{\$ 2 . 0 0}$ (if you indicate 100 chances in 100 for the cup that was used), and a low payoff of $\mathbf{\$ 0 . 0 0}$ (if you indicate 0 chances in 100 for the cup that was used.)

Payoff Table: Please look at the payoff table below. The top row on the left side indicates that if you report 0 chances in 100 for the Red Cup, you will earn $\$ 0.00$ if the Red Cup was used and \$2.00 if the Blue Cup was used. Conversely, the bottom row on the right indicates that if your report 100 chances in 100 for the Red Cup, you will earn $\$ 2.00$ if the Red Cup was used and \$0.00 if the Blue Cup was used.

Payoff Formula: The payoffs in the table are constructed by starting with a base amount \$2.00 and subtracting a penalty that depends on how much probability you assign to the cup, Red or Blue, that is NOT used. (This penalty is 2 times the square of the probability assigned to the Cup that was NOT used.) In any case, the calculations are done for you in the table.

$\begin{array}{llllll}\begin{array}{l}\text { Reported } \\ \text { Chances of }\end{array} & \begin{array}{l}\text { Payoff if } \\ \text { Red Cup }\end{array} & \begin{array}{l}\text { Payoff if } \\ \text { Blue Cup }\end{array} & \begin{array}{l}\text { Reported } \\ \text { Chances of }\end{array} & \begin{array}{l}\text { Payoff if } \\ \text { Red Cup }\end{array} & \begin{array}{l}\text { Payoff if } \\ \text { Blue Cup }\end{array} \\ \text { Red Cup } & \text { was used } & \text { was used } & \text { Red Cup } & \text { was used } & \text { was used } \\ \mathbf{0} & \$ 0.00 & \$ 2.00 & 50 & \$ 1.50 & \$ 1.50 \\ 1 & \$ 0.04 & \$ 2.00 & 51 & \$ 1.52 & \$ 1.48 \\ 2 & \$ 0.08 & \$ 2.00 & 52 & \$ 1.54 & \$ 1.46 \\ 3 & \$ 0.12 & \$ 2.00 & 53 & \$ 1.56 & \$ 1.44\end{array}$




\begin{tabular}{|c|c|c|c|c|c|}
\hline 4 & \$0.16 & $\$ 2.00$ & 54 & $\$ 1.58$ & $\$ 1.42$ \\
\hline 5 & $\$ 0.20$ & $\$ 2.00$ & 55 & $\$ 1.60$ & $\$ 1.40$ \\
\hline 6 & $\$ 0.23$ & $\$ 1.99$ & 56 & $\$ 1.61$ & $\$ 1.37$ \\
\hline 7 & $\$ 0.27$ & $\$ 1.99$ & 57 & $\$ 1.63$ & $\$ 1.35$ \\
\hline 8 & $\$ 0.31$ & $\$ 1.99$ & 58 & $\$ 1.65$ & $\$ 1.33$ \\
\hline 9 & $\$ 0.34$ & $\$ 1.98$ & 59 & $\$ 1.66$ & $\$ 1.30$ \\
\hline 10 & $\$ 0.38$ & $\$ 1.98$ & 60 & $\$ 1.68$ & $\$ 1.28$ \\
\hline 11 & $\$ 0.42$ & $\$ 1.98$ & 61 & $\$ 1.70$ & $\$ 1.26$ \\
\hline 12 & $\$ 0.45$ & $\$ 1.97$ & 62 & $\$ 1.71$ & $\$ 1.23$ \\
\hline 13 & $\$ 0.49$ & $\$ 1.97$ & 63 & $\$ 1.73$ & $\$ 1.21$ \\
\hline 14 & $\$ 0.52$ & $\$ 1.96$ & 64 & $\$ 1.74$ & $\$ 1.18$ \\
\hline 15 & $\$ 0.56$ & $\$ 1.96$ & 65 & $\$ 1.76$ & $\$ 1.15$ \\
\hline 16 & $\$ 0.59$ & $\$ 1.95$ & 66 & $\$ 1.77$ & $\$ 1.13$ \\
\hline 17 & $\$ 0.62$ & $\$ 1.94$ & 67 & $\$ 1.78$ & $\$ 1.10$ \\
\hline 18 & $\$ 0.66$ & $\$ 1.94$ & 68 & $\$ 1.80$ & $\$ 1.08$ \\
\hline 19 & $\$ 0.69$ & $\$ 1.93$ & 69 & $\$ 1.81$ & $\$ 1.05$ \\
\hline 20 & $\$ 0.72$ & $\$ 1.92$ & 70 & $\$ 1.82$ & $\$ 1.02$ \\
\hline 21 & $\$ 0.75$ & $\$ 1.91$ & 71 & $\$ 1.83$ & $\$ 0.99$ \\
\hline 22 & $\$ 0.78$ & $\$ 1.90$ & 72 & $\$ 1.84$ & $\$ 0.96$ \\
\hline 23 & $\$ 0.81$ & $\$ 1.89$ & 73 & $\$ 1.85$ & $\$ 0.93$ \\
\hline 24 & $\$ 0.84$ & $\$ 1.88$ & 74 & $\$ 1.86$ & $\$ 0.90$ \\
\hline 25 & $\$ 0.88$ & $\$ 1.88$ & 75 & $\$ 1.88$ & $\$ 0.88$ \\
\hline 26 & $\$ 0.90$ & $\$ 1.86$ & 76 & $\$ 1.88$ & $\$ 0.84$ \\
\hline 27 & $\$ 0.93$ & $\$ 1.85$ & 77 & $\$ 1.89$ & $\$ 0.81$ \\
\hline 28 & $\$ 0.96$ & $\$ 1.84$ & 78 & $\$ 1.90$ & $\$ 0.78$ \\
\hline
\end{tabular}




\begin{tabular}{|c|c|c|c|c|c|}
\hline 29 & $\$ 0.99$ & $\$ 1.83$ & 79 & $\$ 1.91$ & $\$ 0.75$ \\
\hline 30 & $\$ 1.02$ & $\$ 1.82$ & 80 & $\$ 1.92$ & \$0.72 \\
\hline 31 & $\$ 1.05$ & $\$ 1.81$ & 81 & $\$ 1.93$ & $\$ 0.69$ \\
\hline 32 & $\$ 1.08$ & $\$ 1.80$ & 82 & $\$ 1.94$ & $\$ 0.66$ \\
\hline 33 & $\$ 1.10$ & $\$ 1.78$ & 83 & $\$ 1.94$ & $\$ 0.62$ \\
\hline 34 & $\$ 1.13$ & $\$ 1.77$ & 84 & $\$ 1.95$ & $\$ 0.59$ \\
\hline 35 & $\$ 1.15$ & $\$ 1.76$ & 85 & $\$ 1.96$ & $\$ 0.56$ \\
\hline 36 & $\$ 1.18$ & $\$ 1.74$ & 86 & $\$ 1.96$ & \$0.52 \\
\hline 37 & $\$ 1.21$ & $\$ 1.73$ & 87 & $\$ 1.97$ & $\$ 0.49$ \\
\hline 38 & $\$ 1.23$ & $\$ 1.71$ & 88 & $\$ 1.97$ & $\$ 0.45$ \\
\hline 39 & $\$ 1.26$ & $\$ 1.70$ & 89 & $\$ 1.98$ & $\$ 0.42$ \\
\hline 40 & $\$ 1.28$ & $\$ 1.68$ & 90 & $\$ 1.98$ & $\$ 0.38$ \\
\hline 41 & $\$ 1.30$ & $\$ 1.66$ & 91 & $\$ 1.98$ & $\$ 0.34$ \\
\hline 42 & $\$ 1.33$ & $\$ 1.65$ & 92 & $\$ 1.99$ & $\$ 0.31$ \\
\hline 43 & $\$ 1.35$ & $\$ 1.63$ & 93 & $\$ 1.99$ & $\$ 0.27$ \\
\hline 44 & $\$ 1.37$ & $\$ 1.61$ & 94 & $\$ 1.99$ & $\$ 0.23$ \\
\hline 45 & $\$ 1.40$ & $\$ 1.60$ & 95 & $\$ 2.00$ & $\$ 0.20$ \\
\hline 46 & $\$ 1.42$ & $\$ 1.58$ & 96 & $\$ 2.00$ & $\$ 0.16$ \\
\hline 47 & $\$ 1.44$ & $\$ 1.56$ & 97 & $\$ 2.00$ & $\$ 0.12$ \\
\hline 48 & $\$ 1.46$ & $\$ 1.54$ & 98 & $\$ 2.00$ & \$0.08 \\
\hline 49 & $\$ 1.48$ & $\$ 1.52$ & 99 & $\$ 2.00$ & $\$ 0.04$ \\
\hline 50 & $\$ 1.50$ & $\$ 1.50$ & 100 & $\$ 2.00$ & $\$ 0.00$ \\
\hline Chances of & Payoff if & Payoff if & Chances of & Payoff if & Payoff if \\
\hline Red Cup & Red Cup & Blue Cup & Red Cup & Red Cup & Blue Cup \\
\hline
\end{tabular}


Making Your Decision: In making your decision, you should think carefully about the chances out of 100 that the Red Cup is being used, since making a good decision based on the available information will help you earn more money. If your decision represents your true beliefs about the chances that the red cup is being used, then your expected earnings (determined by multiplying probabilities times corresponding payoffs) will be maximized.

\section{Summary (Final Page of Instructions)}

\section{Blue Cup Red Cup}

(if die throw is 1 - 3) (if die throw is 4 - 6)

\section{B B R R R B}

Cup Used: If the roll of the die yields 1 - 3, then the draw will be from the Blue cup, which contains more blue marbles. If the roll of the die yields $4-6$, then the draw will be from the Red cup, which contains more red marbles. Note that each cup is equally likely to be used.

Information: After the cup to be used has been randomly determined, you will be shown sample draws, if any, from that cup (made with replacement) for the current round. After considering the available information, you enter your decision (an integer between 0 and 100) in the box provided.

Payment Method: After you have submitted your "chances in 100" that the Red Cup is being used, you will be informed about which cup was actually used. You will earn more money to the extent to which your decision indicated higher chances in 100 for the cup that turned out to be the one that actually was used, as shown by the payoff table shown below.

Helpful Hint: Your decision should be your best guess about the chances out of 100 that the Red Cup is being used; this decision will maximize your expected earnings.

Final Hint: After you look at the draws (if any) for a round, try to figure out which cup, Red or Blue, is more likely, and be sure that your decision reflects that 
intuition, i.e. that the chances in 100 for the Red Cup are greater than 50 if you think the Red Cup is more likely and less than 50 otherwise.

\section{QSR-numbers Treatment Instructions (pages 3-6)}

When you have seen the draws (if any), you will select a row from a payoff table with rows that are numbered from 0 to 100 .

Lower numbered rows provide lower money payoffs if the Red Cup was used (and higher payoffs if the Blue Cup was used).

Conversely, rows with higher numbers provide higher payoffs if the Red Cup was used (and lower payoffs if the Blue Cup was used).

Payment Method: After you have selected a row from the table, you will be informed about which cup was actually used, which determines the relevant payoff from the row you previously selected.

Payoff Table: Please look at the payoff table below. The top row on the left side indicates that if you choose row 0 , you will earn $\$ 0.00$ if the Red Cup was used and $\$ 2.00$ if the Blue Cup was used. Conversely, the bottom row on the right indicates that if your choose row 100 , you will earn $\$ 2.00$ if the Red Cup was used and $\$ 0.00$ if the Blue Cup was used.

Your Choice: You are free to select any row number you want. Note that there is no correct or incorrect choice. The rows selected may may differ from one individual to the next. In general, however, you may find it profitable to choose a higher row number when you think the chances that the Red Cup was used are higher. Indeed, a higher row number will provide a higher money payoff if the Red Cup was used. Conversely, you may find it profitable to choose a lower row number when you think the chances that the Red Cup was used are lower.

\begin{tabular}{|c|c|c|c|c|c|}
\hline $\begin{array}{c}\text { Row } \\
\text { Number }\end{array}$ & $\begin{array}{l}\text { Payoff if } \\
\text { Red Cup } \\
\text { was used }\end{array}$ & $\begin{array}{l}\text { Payoff if } \\
\text { Blue Cup } \\
\text { was used }\end{array}$ & $\begin{array}{c}\text { Row } \\
\text { Number }\end{array}$ & $\begin{array}{l}\text { Payoff if } \\
\text { Red Cup } \\
\text { was used }\end{array}$ & $\begin{array}{l}\text { Payoff if } \\
\text { Blue Cup } \\
\text { was used }\end{array}$ \\
\hline 0 & $\$ 0.00$ & $\$ 2.00$ & 50 & $\$ 1.50$ & $\$ 1.50$ \\
\hline
\end{tabular}




\begin{tabular}{|c|c|c|c|c|c|}
\hline 1 & $\$ 0.04$ & $\$ 2.00$ & 51 & $\$ 1.52$ & $\$ 1.48$ \\
\hline 2 & $\$ 0.08$ & $\$ 2.00$ & 52 & $\$ 1.54$ & $\$ 1.46$ \\
\hline 3 & $\$ 0.12$ & $\$ 2.00$ & 53 & $\$ 1.56$ & $\$ 1.44$ \\
\hline 4 & $\$ 0.16$ & $\$ 2.00$ & 54 & $\$ 1.58$ & $\$ 1.42$ \\
\hline 5 & $\$ 0.20$ & $\$ 2.00$ & 55 & $\$ 1.60$ & $\$ 1.40$ \\
\hline 6 & $\$ 0.23$ & $\$ 1.99$ & 56 & $\$ 1.61$ & $\$ 1.37$ \\
\hline 7 & $\$ 0.27$ & $\$ 1.99$ & 57 & $\$ 1.63$ & $\$ 1.35$ \\
\hline 8 & $\$ 0.31$ & $\$ 1.99$ & 58 & $\$ 1.65$ & $\$ 1.33$ \\
\hline 9 & $\$ 0.34$ & $\$ 1.98$ & 59 & $\$ 1.66$ & $\$ 1.30$ \\
\hline 10 & $\$ 0.38$ & $\$ 1.98$ & 60 & $\$ 1.68$ & $\$ 1.28$ \\
\hline 11 & $\$ 0.42$ & $\$ 1.98$ & 61 & $\$ 1.70$ & $\$ 1.26$ \\
\hline 12 & $\$ 0.45$ & $\$ 1.97$ & 62 & $\$ 1.71$ & $\$ 1.23$ \\
\hline 13 & $\$ 0.49$ & $\$ 1.97$ & 63 & $\$ 1.73$ & $\$ 1.21$ \\
\hline 14 & $\$ 0.52$ & $\$ 1.96$ & 64 & $\$ 1.74$ & $\$ 1.18$ \\
\hline 15 & $\$ 0.56$ & $\$ 1.96$ & 65 & $\$ 1.76$ & $\$ 1.15$ \\
\hline 16 & $\$ 0.59$ & $\$ 1.95$ & 66 & $\$ 1.77$ & $\$ 1.13$ \\
\hline 17 & $\$ 0.62$ & $\$ 1.94$ & 67 & $\$ 1.78$ & $\$ 1.10$ \\
\hline 18 & $\$ 0.66$ & $\$ 1.94$ & 68 & $\$ 1.80$ & $\$ 1.08$ \\
\hline 19 & $\$ 0.69$ & $\$ 1.93$ & 69 & $\$ 1.81$ & $\$ 1.05$ \\
\hline 20 & $\$ 0.72$ & $\$ 1.92$ & 70 & $\$ 1.82$ & $\$ 1.02$ \\
\hline 21 & $\$ 0.75$ & $\$ 1.91$ & 71 & $\$ 1.83$ & $\$ 0.99$ \\
\hline 22 & $\$ 0.78$ & $\$ 1.90$ & 72 & $\$ 1.84$ & $\$ 0.96$ \\
\hline 23 & $\$ 0.81$ & $\$ 1.89$ & 73 & $\$ 1.85$ & $\$ 0.93$ \\
\hline 24 & $\$ 0.84$ & $\$ 1.88$ & 74 & $\$ 1.86$ & $\$ 0.90$ \\
\hline 25 & $\$ 0.88$ & $\$ 1.88$ & 75 & $\$ 1.88$ & $\$ 0.88$ \\
\hline
\end{tabular}




\begin{tabular}{|c|c|c|c|c|c|}
\hline 26 & $\$ 0.90$ & $\$ 1.86$ & 76 & $\$ 1.88$ & $\$ 0.84$ \\
\hline 27 & $\$ 0.93$ & $\$ 1.85$ & 77 & $\$ 1.89$ & $\$ 0.81$ \\
\hline 28 & $\$ 0.96$ & $\$ 1.84$ & 78 & $\$ 1.90$ & $\$ 0.78$ \\
\hline 29 & $\$ 0.99$ & $\$ 1.83$ & 79 & $\$ 1.91$ & $\$ 0.75$ \\
\hline 30 & $\$ 1.02$ & $\$ 1.82$ & 80 & $\$ 1.92$ & \$0.72 \\
\hline 31 & $\$ 1.05$ & $\$ 1.81$ & 81 & $\$ 1.93$ & $\$ 0.69$ \\
\hline 32 & $\$ 1.08$ & $\$ 1.80$ & 82 & $\$ 1.94$ & $\$ 0.66$ \\
\hline 33 & $\$ 1.10$ & $\$ 1.78$ & 83 & $\$ 1.94$ & $\$ 0.62$ \\
\hline 34 & $\$ 1.13$ & $\$ 1.77$ & 84 & $\$ 1.95$ & $\$ 0.59$ \\
\hline 35 & $\$ 1.15$ & $\$ 1.76$ & 85 & $\$ 1.96$ & $\$ 0.56$ \\
\hline 36 & $\$ 1.18$ & $\$ 1.74$ & 86 & $\$ 1.96$ & $\$ 0.52$ \\
\hline 37 & $\$ 1.21$ & $\$ 1.73$ & 87 & $\$ 1.97$ & $\$ 0.49$ \\
\hline 38 & $\$ 1.23$ & $\$ 1.71$ & 88 & $\$ 1.97$ & $\$ 0.45$ \\
\hline 39 & $\$ 1.26$ & $\$ 1.70$ & 89 & $\$ 1.98$ & $\$ 0.42$ \\
\hline 40 & $\$ 1.28$ & $\$ 1.68$ & 90 & $\$ 1.98$ & $\$ 0.38$ \\
\hline 41 & $\$ 1.30$ & $\$ 1.66$ & 91 & $\$ 1.98$ & $\$ 0.34$ \\
\hline 42 & $\$ 1.33$ & $\$ 1.65$ & 92 & $\$ 1.99$ & \$0.31 \\
\hline 43 & $\$ 1.35$ & $\$ 1.63$ & 93 & $\$ 1.99$ & \$0.27 \\
\hline 44 & $\$ 1.37$ & $\$ 1.61$ & 94 & $\$ 1.99$ & $\$ 0.23$ \\
\hline 45 & $\$ 1.40$ & $\$ 1.60$ & 95 & $\$ 2.00$ & $\$ 0.20$ \\
\hline 46 & $\$ 1.42$ & $\$ 1.58$ & 96 & $\$ 2.00$ & $\$ 0.16$ \\
\hline 47 & $\$ 1.44$ & $\$ 1.56$ & 97 & $\$ 2.00$ & \$0.12 \\
\hline 48 & $\$ 1.46$ & $\$ 1.54$ & 98 & $\$ 2.00$ & $\$ 0.08$ \\
\hline 49 & $\$ 1.48$ & $\$ 1.52$ & 99 & $\$ 2.00$ & $\$ 0.04$ \\
\hline 50 & $\$ 1.50$ & $\$ 1.50$ & 100 & $\$ 2.00$ & $\$ 0.00$ \\
\hline
\end{tabular}




\section{Row Payoff if Payoff if Row Payoff if Payoff if Number Red Cup Blue Cup Number Red Cup Blue Cup}

Making Your Decision: In making your payoff row decision, you should think carefully about the chances that the Red Cup is being used, since making a good decision based on the available information will help you earn more money. 


\section{Summary (Final Page of Instructions)}

\section{Blue Cup $\quad$ Red Cup}

(if die throw is 1 - 3) (if die throw is $4-6$ )

\section{B B R $\quad$ R R B}

Cup Used: If the roll of the die yields 1 - 3, then the draw will be from the Blue cup, which contains more blue marbles. If the roll of the die yields 4 - 6 , then the draw will be from the Red cup, which contains more red marbles. Note that each cup is equally likely to be used.

Information: After the cup to be used has been randomly determined, you will be shown sample draws, if any, from that cup (made with replacement) for the current round. After considering the available information, you enter your row choice (an integer between 0 and 100) in the box provided.

Payment Method: After you have submitted your row choice, you will be informed about which cup was actually used, and your payoff will be calculated from the relevant row of the table below.

Helpful Hint: After you look at the draws (if any) for a round, try to figure out which cup, Red or Blue, is more likely, and be sure that your decision reflects that intuition, i.e. you should consider choosing a row of 50 or above if you believe that the chances for the Red Cup are greater, and you should consider choosing a row of 50 or lower if you think the chances for the Blue Cup are greater. 